\title{
Plurilinguisme littéraire : de la théorie à la genèse
}

\section{Emilio Sciarrino et Olga Anokhina}

\section{(2) OpenEdition}

Journals

Édition électronique

URL : https://journals.openedition.org/genesis/2554

DOI : 10.4000/genesis.2554

ISSN : 2268-1590

\section{Éditeur :}

Presses universitaires de Paris Sorbonne (PUPS), Société internationale de génétique artistique littéraire et scientifique (SIGALES)

\section{Édition imprimée}

Date de publication : 4 juin 2018

Pagination : 11-34

ISBN : 979-10-231-0604-6

ISSN : 1167-5101

\section{Référence électronique}

Emilio Sciarrino et Olga Anokhina, «Plurilinguisme littéraire : de la théorie à la genèse », Genesis [En

ligne], 46 | 2018, mis en ligne le 01 juin 2019, consulté le 17 août 2022. URL : http://

journals.openedition.org/genesis/2554; DOI : https://doi.org/10.4000/genesis.2554 


\title{
Plurilinguisme littéraire : de la théorie à la genèse
}

\author{
Olga Anokhina et Emilio Sciarrino
}

$\mathrm{D}$ epuis les dernières décennies, les chercheurs s'intéressent de plus près aux enjeux du plurilinguisme dans plusieurs domaines scientifiques. Cependant, rares sont les recherches qui interrogent le lien entre le plurilinguisme et la créativité dans le domaine de l'écrit. Pourtant, à la suite de mouvements migratoires toujours croissants depuis le siècle dernier, de nombreux écrivains plurilingues ont émergé dans différents états européens (en France, en Allemagne, en Grèce, en Italie, en Belgique, dans les pays nordiques ou encore dans les pays de l'ex-Union soviétique, etc.). Un certain nombre de ces écrivains qui créent dans la langue de leur pays d'adoption ont bénéficié d'une reconnaissance par la critique, par le grand public et par ce qu'on peut appeler l'establishment culturel. Ainsi, de nombreux écrivains qui ont adopté l'allemand pour leur écriture ont reçu des prix très prestigieux en Allemagne' ${ }^{1}$. La France a également distingué plusieurs écrivains d'origine étrangère, dont les cas les plus connus sont sans doute François Cheng et Andréï Makine, tous les deux lauréats de nombreux prix littéraires et élus à l'Académie française 2 . Or, ces écrivains pleinement reconnus par leur pays d'adoption ne représentent que la pointe visible de l'iceberg, car le nombre important des auteurs qui vivent et écrivent entre les langues ${ }^{3}$ remet en cause l'idée selon laquelle il s'agirait d'un phénomène marginal ou exceptionnel.

Par ailleurs, il convient de rappeler que le plurilinguisme littéraire n'est pas un fait nouveau qui se limiterait aux XXe et XXIe siècles. Au contraire, il représente une tradition prégnante dans les littératures occidentales depuis le Moyen Âge, comme l'a montré Leonard Forster dans son essai pionnier ${ }^{4}$. Mais les premières manifestations de l'écriture plurilingue sont encore plus anciennes et remontent à l'Antiquité. On peut citer évidemment la célèbre pierre de Rosette (196 avant J.-C) qui présente - sur le même support - trois versions d'un même texte : égyptien en hiéroglyphes, égyptien démotique et alphabet grec. De nombreux documents de l'Antiquité tardive témoignent également de la coexistence de plusieurs langues au sein

1. Dirk Weissmann, «Von Özdamar zu Petrowskaja und weiter. Interkulturelle Literatur und Literaturpreise im deutschsprachigen Raum unter besonderer Berücksichtigung des Ingeborg Bachmann-Preises », Études Germaniques, $\mathrm{n}^{\circ}$ 287, Éditions Klincksieck, 2017, p. 337-352.

2. Cette reconnaissance ne fait que confirmer un phénomène existant : faut-il rappeler que des écrivains français reconnus comme Henri Troyat ou Romain Gary sont d'origine étrangère également. On peut aussi mentionner la vague d'émigration des artistes lors de la guerre civile en Espagne ou celle des écrivains latino-américains qui se sont installés dans la ville qu'ils considéraient comme la capitale culturelle du monde - Paris - au moment de l'installation des régimes dictatoriaux dans leurs pays d'origine.

3. Olga Anokhina et Alain Ausoni (dir.), Vivre entre les langues, écrire en français, Paris, Éditions des archives contemporaines, coll. «Multilinguisme, traduction, création», 2018 (à paraître).

4. Leonard Forster, The Poet's Tongues: Multilingualism in Literature, Otago-Cambridge, Cambridge University Press-University of Otago Press, 1970. Voir aussi la synthèse de A. Knauth, «Literary Multilingualism I: General Outlines and Western World», dans M. Seligmann-Silva et al. (dir.), Comparative literature: sharing knowledges for preserving cultural diversity, Oxford, Eolss Publishers : <http://www.eolss.net/ebooks/sample\%20chapters/c04/ e6-87-07-05.pdf > (consulté en décembre 2017). 
des documents écrits de différentes natures (juridique, littéraire, correspondance) 5 . La poésie

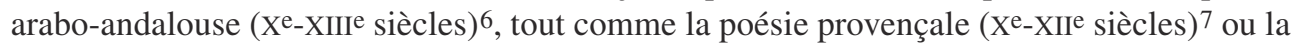
poésie médiévale basque (XVe et XVIe siècles) ${ }^{8}$ sont autant d'exemples du plurilinguisme qui trouvent leur manifestation dans la production littéraire européenne. Sur un autre continent et dans une culture très différente, on constate des phénomènes de plurilinguisme dans les écrits du peuple maya et notamment dans le document appelé le Codex de Madrid (el Códice de Madrid). Les chercheurs ont pu y identifier le mélange des langues mayas (englobées sous le nom de yucatèque) avec la famille de langues cholanes 9 .

Nombreux sont les chefs-d'œuvre de la littérature européenne qui font du plurilinguisme un des principes essentiels de leur poétique : pensons à Dante ou encore à Joyce, pour ne citer que deux exemples célèbres. Aux grands classiques plurilingues consacrés par la critique on pourra ajouter également le vaste domaine de la littérature postcoloniale ${ }^{10}$; le corpus des écrivains migrants 11 et «exilés »; l'ensemble, assez peu représenté en France, mais important dans d'autres pays, des écrivains utilisant un dialecte ${ }^{12}$; ou encore tous les auteurs qui retranscrivent dans leurs œuvres des situations de bilinguisme ou de diglossie ${ }^{13}$; sans compter enfin les écrivains polyglottes qui, pour d'innombrables raisons, y compris des choix

5. Voir le cycle de conférences en ligne de Jean-Luc Fournet au Collège de France : Jean-Luc Fournet, Multilinguisme et multiculturalisme dans l'Égypte de l'Antiquité tardive, cours au Collège de France en 2016 et $2017:<$ http:// www.college-de-france.fr/site/jean-luc-fournet/index.htm > (consulté en décembre 2017).

6. Alexandre Koudéline, «La poésie strophique arabo-hispanique : le cas particulier du multilinguisme médiéval», dans Olga Anokhina, Natalia Vélikanova et al. (dir.), Multilinguisme et genèse des textes, Moscou, Nauka, 2010, p. 24-31. L'auteur attire notre attention sur le fait que la connaissance de plusieurs langues n'était pas à cette époque le privilège d'un cercle restreint des savants et qu'une partie importante de population andalouse était bilingue voire trilingue.

7. À ce sujet, voir l'article de Rainier Grutman, «Le système triplement bilingue de la lyrique occitane», dans Revues des langues romanes, $\mathrm{n}^{\circ}$ 2, tome XCVIII, 1994, p. 465-475. Un exemple remarquable d'écriture plurilingue est le poème «eras quan vey verdeyar» de Raimbaut de Vaqueiras composé en cinq langues : occitan, français, italien, gascon, gallicien.

8. «Canción medieval vasca» dans Francisco Sensio Barbieri, Cancionero musical de los siglos XV y XVI, Madrid, Academia de Bellas Artes de san Fernando, 1890, Jesús María De Leizaola, Estudios Sobre La Poesía Vasca, Buenos Aires, 1951, p. 27.

9. Alfonso Lacadena, «Bilingüismo en el Códice de Madrid», Los Investigadores de la Cultura Maya, México, Publicaciones de la Universidad Autónoma de Campeche, n 5, 1997, p. 184-204 et Victoria Bricker, «Bilingualism in the Maya Codices and the Books of Chilam Balam», Language and Dialect Variation in the Maya Hieroglyphic Script, edited by Gabrielle Vail and Martha J. Macri. Special Issue of Written Language and Literacy, volume 3, $\mathrm{n}^{\circ}$ 1, Tulane University, John Benjamins Publishing Company, 2000, p. 77-115.

10. Bill Ashcroft, Gareth Griffiths, Helen Tiffin, The Empire writes back. Theory and Practice in Post-colonial Literatures, London, Routledge, 1989.

11. En ce qui concerne le domaine de langue française, voir Ursula Mathis Moser et Birgit Mertz-Baumgartner (dir.), Passages et ancrages en France. Dictionnaire des écrivains migrants de langue française (1981-2011), Paris, Honoré Champion, 2012, 965 p.

12. C'est le cas de nombreux écrivains italiens, de Pier Paolo Pasolini à Stefano D'Arrigo en passant par Carlo Emilio Gadda.

13. La diglossie indique la coexistence chez un même locuteur et sur le même territoire de deux langues qui ont une utilisation différenciée liée à un contexte social de domination d'une langue sur une autre. Henri Giordan et Alain Ricard (dir.), Diglossie et littérature, Bordeaux, Maison des sciences de l'homme d'Aquitaine, 1976; voir aussi à propos de l'usage du terme «diglossie», Myriam Suchet, Outils pour une traduction postcoloniale, Paris, Éditions des archives contemporaines, 2009, p. 43. 
personnels, ont fait de la diversité des langues un de leurs principes créateurs 14 . Enfin, cette liste déjà longue comprend aussi les nombreux cas d'écrivains qui, étant considérés à tort comme monolingues, ont en fait employé différentes langues dans leur processus créatif : nous en verrons plus loin quelques exemples.

Un autre domaine de recherche reste pratiquement inexploré à ce jour : l'étude des artistes plurilingues (peintres ${ }^{15}$, musiciens, plasticiens, photographes, cinéastes...) et de l'impact que leur plurilinguisme exerce sur leur créativité, comme l'illustre l'exemple de Raoul Hausmann présenté dans ce numéro.

Malgré la richesse et la diversité de ce patrimoine littéraire mondial, une certaine vision traditionnellement monolingue de l'écriture a longtemps entravé la prise en considération du plurilinguisme. Une telle conception biaisée, associant une langue et une littérature à une nation, est en fait héritée des subdivisions du savoir occidental forgées au XIXe siècle, qui ont été depuis largement relativisées ${ }^{16}$, mais qui continuent néanmoins d'influencer lourdement les dynamiques de réception contemporaines.

C'est pourquoi, en dépit des exemples de plurilinguisme littéraire que nous venons de citer, il aura fallu que de nombreuses études s'efforcent de déconstruire l'idée du monolinguisme, encore prégnante dans le champ scientifique ${ }^{17}$, afin de constituer progressivement un véritable champ d'études aujourd'hui émergent, dont les enjeux ne sont pas seulement esthétiques ou littéraires au sens strict, mais aussi philosophiques et anthropologiques ${ }^{18}$. Cependant, face à la multiplication exponentielle d'approches divergentes et en raison de la forte dispersion des terminologies critiques et des systèmes conceptuels mobilisés, il est nécessaire d'engager une réflexion méthodologique et épistémologique. Dans ce contexte, la critique génétique joue un rôle central, en remettant au cœur de l'étude les textes et les avant-textes qui permettent d'explorer les processus de création plurilingue, afin d'en tirer des conclusions théoriques.

Quand il s'agit d'une discipline jeune, ce qui est le cas des recherches dans le domaine du plurilinguisme littéraire, la question de la terminologie se pose très souvent. En effet, comment désigner ce phénomène d'écriture en plusieurs langues? On peut emprunter à la sociolinguistique la notion du code-switching qui désigne une alternance de deux ou plusieurs langues ou dialectes au sein du même acte énonciatif. Ce concept a été élaboré pour rendre compte des changements de langues très fréquents au sein du même discours oral chez des sujets plurilingues. Or, on observe des phénomènes très similaires à l'écrit. Cependant, comme nous le verrons, il semble

14. Voir Akira Mizubayashi, Une langue venue d'ailleurs, Paris, Gallimard, 2011.

15. Par exemple, Natasha Lvovich démontre comment le plurilinguisme de Chagall se manifeste dans ses créations picturales et notamment celle de The Self-Portrait with Seven Fingers [Autoportrait aux 7 doigts]. Cf. Natasha Lvovich, «Translingual Identity and Art: Marc Chagall's Stride through the Gates of Janus », dans Critical Multilingualism Studies, ${ }^{\circ}$ 3/1, 2015, p. 112-134. Disponible en ligne à l'adresse suivante : < http://cms.arizona. edu/index.php/multilingual/article/download/57/108> (consulté en décembre 2017).

16. Sur la critique des littératures nationales, voir Ernst Robert Curtius, La Littérature européenne et le Moyen Âge latin, traduit par Jean Bréjoux, Paris, Presses universitaires de France, 1956, p. 24.

17. Aneta Pavlenko, Emotions and Multilingualism, Cambridge, Cambridge University Press, 2005, p. 3.

18. Barbara Cassin, Éloge de la traduction. Compliquer l'universel, Paris, Fayard, 2016 et Fabienne Durand-Bogaert, entretien avec Barbara Cassin «Il faut au moins deux langues pour savoir qu'on en parle une », Genesis, $\mathrm{n}^{\circ} 38$, «Traduire», 2014, p. 129-137.

\section{Flou terminologique et conceptuel}


judicieux de désigner par ce terme l'une des stratégies créatives que nous allons identifier et qui fait place, lors de l'écriture et/ou dans une œuvre publiée, à plus d'une langue 19.

Nous pourrions également utiliser la notion d'hétérolinguisme proposée par Rainier Grutman pour signaler spécifiquement des phénomènes hétérolingues observés dans l'écriture. L'hétérolinguisme désigne «la présence dans un texte d'idiomes étrangers, sous quelque forme que ce soit, aussi bien que de variétés (sociales, régionales ou chronologiques) de la langue principale $20_{»}$. En effet, le terme hétérolingue présente une alternative synonymique heureuse au terme «plurilingue» qu'on discutera plus loin.

Certains chercheurs nord-américains préfèrent, quant à eux, parler du translinguisme. Ce terme a été popularisé par les travaux de Steven G. Kellman ${ }^{21}$. Selon lui, les écrivains translingues sont des auteurs qui écrivent en plus d'une langue, ou bien dans une langue autre que leur langue maternelle, ou bien dans un mélange de langues. Il nous semble que ce terme, adopté également par certains chercheurs européens, n'apporte aucune valeur ajoutée - du moins dans sa définition évoquée plus haut - et ne fait que renforcer le flou conceptuel et terminologique qui règne dans ce domaine à l'heure actuelle, d'autant plus qu'il n'est pas situé par rapport aux concepts déjà existants. Le terme de translingueltranslinguisme provient en effet de la langue anglaise (translingual/transilingualism) et entre en concurrence avec les concepts utilisés dans les langues romanes (multilingue/multilinguisme vs plurilingue/ plurilinguisme). Cependant, ce terme peut être quelquefois pertinent quand il désigne des phénomènes linguistiques singuliers qui apparaissent à la suite de l'interaction de plusieurs langues. Ce type de phénomène est parfaitement illustré dans ce numéro par la contribution de Dirk Weissmann sur Paul Celan. Il s'agit dans ce cas non pas d'écrivains mais bien de manifestations, de phénomènes textuels translingues.

De nombreux chercheurs européens, et notamment les chercheurs français, ont choisi de suivre les recommandations européennes dans un souci d'harmonisation terminologique 22 . Le multilinguisme renvoie alors à la coexistence de plusieurs langues sur un même territoire, au sein d'un même pays, au cœur d'une société. Par définition, un pays ou une communauté sera multilingue. Le plurilinguisme, quant à lui, est relatif à l'individu. Il définit la capacité d'une personne à maîtriser plusieurs langues. Ainsi, même certains chercheurs anglo-saxons - rares il est vrai, mais particulièrement éclairés -, comme William Mackey, auquel on doit un apport important aux études du plurilinguisme pendant plusieurs décennies ${ }^{23}$, militent pour l'emploi du terme plurilinguisme pour désigner des individus, y compris dans la langue anglaise :

In the vast literature on bilingualism there is really no consensus on what the term means. [...] Bilingualism can be seen as a double continuum of mutually modifying practices including degrees of use and competence in each of the languages coupled with degrees of difference of them. [...] The

19. Nous définissons les stratégies d'écriture comme les différentes pratiques adoptées par les scripteurs (consciemment ou inconsciemment) pour arriver à tel ou tel objectif (en l'occurrence à la rédaction d'un texte). 20. Rainier Grutman, Des langues qui résonnent. L'hétérolinguisme au XIXe siècle québécois, Montréal, Fides-CETUQ, 1997, p. 37.

21. Steven Kellman, The translingual imagination, Lincoln, University of Nebraska Press, 2000 et Steven Kellman, Switching Languages: Translingual Writers Reflect on Their Craft, Lincoln/London, University of Nebraska Press, 2003.

22. Conseil de l'Europe, <http://www.coe.int/t/dg4/linguistic/Division_FR.asp > (consulté en décembre 2017).

23. William Mackey, Bilinguisme et contact des langues, Paris, Éditions Klincksieck, 1976. 
variables of bilingualism are equally applicable to the use of three or more languages, that is, to cases of multilingualism [...] since they are numerical extensions of the same variable [...]. It is for such reasons perhaps that, during the past decades, European publications have opted for a more general term which subsumes both bilingualism and multilingualism. That is why the term plurilingualism (Fr. plurilinguisme; Gr. Mehrsprachigkeit) for more then one language seems to be gaining ground $[\ldots]$. This trend will appear in the following pages whenever the term might seem preferable 24 .

On constate donc qu'aujourd'hui la terminologie n'est pas fixée - si elle doit l'être un jour - et qu'il existera toujours des difficultés pour trancher entre les termes multilingues versus plurilingues notamment concernant les textes, comme le souligne François Rastier :

Nous avions distingué [...] le plurilinguisme (capacité de maîtriser plusieurs langues) du multilinguisme (coexistence de plusieurs langues dans un espace social), mais l'œuvre multilingue relativise, voire dépasse cette distinction devenue provisoire, car si les langues restent juxtaposées au sein du texte empirique, au sein de l'œuvre, elles se parlent à leur manière et parlent diversement au lecteur en tenant un discours commun, quand bien même elles affichent une hétérogénéité de surface 25 .

Ainsi, dans ce numéro nous avons pris le parti de suivre les recommandations et les usages européens en matière terminologique en privilégiant le terme «plurilingue», en référence aux écrivains et à leurs textes 26 . Par ailleurs, il nous semble important de proposer une nouvelle définition de l'écrivain plurilingue, définition qui intègre toutes les données et les caractéristiques que nous allons évoquer dans cette contribution. Nous désignons comme écrivain plurilingue une personne qui, lors de son écriture, utilise au moins deux langues dont on peut trouver les traces - explicites ou implicites - soit dans ses œuvres publiées, soit dans les documents de travail qui accompagnent son processus créatif (les brouillons, les notes, le journal d'écriture, etc.), même si l'œuvre publiée a une apparence monolingue. Par extension, le texte ainsi produit sera également désigné comme plurilingue.

Les études critiques littéraires menées au sujet des écrivains plurilingues depuis les années 2000 en France 27 et à l'étranger 28 ont incontestablement contribué à la prise de conscience de l'ampleur de ce phénomène. Mais ces recherches présentent plusieurs limites. D'une part, dans leur majorité, celles-ci portent presque exclusivement leur attention sur des

\section{Les acquis de la recherche sur le plurilinguisme littéraire}

24. William Mackey, «Bilingualism and Multilingualism in North America», dans Tej K. Bhatia and William C. Ritchie (dir.), The Handbook of Bilingualism and Multilingualism: Second Edition, Oxford, Blackwell Publishing, 2013, p. 707-708.

25. François Rastier, «Introduction», dans Olga Anokhina et François Rastier (dir.), Écrire en langues : littératures et plurilinguisme, Paris, Éditions des archives contemporaines, coll. «Multilinguisme, traduction, création», 2015, p. X.

26. C'est aussi le cas d'Alexis Nouss. Voir le chapitre I de son ouvrage Plaidoyer pour un monde métis, Paris, éditions Textuel, 2005.

27. Voir, à titre d'exemple, Anne-Rosine Delbart, Les Exilés du langage. Un siècle d'écrivains français venus d'ailleurs (1919-2000), Limoges, Presses universitaires de Limoges, coll. «Francophonies», 2005. Axel Gasquet et Modesta Suárez (dir.), Écrivains plurilingues et écritures métisses : l’hospitalité des langues, Clermont-Ferrand, Presses universitaires Blaise Pascal, 2007. Michaël Oustinoff, Bilinguisme d'écriture et autotraduction, Julien Green, Samuel Beckett, Vladimir Nabokov, Paris, L'Harmattan, 2001. Stéphanie Benson, Les Aventuriers de la langue fourchue : l'écriture multilingue et la désintégration de l'espace colonial, thèse de doctorat, université de Bordeaux III, 2011. 28. Yasemin Yildiz, Beyond the Mother Tongue, New York, Fordham University Press, 2012. 
œuvres publiées. Or des œuvres d'apparence monolingue peuvent cacher un processus créatif polyphonique au cours duquel plusieurs langues ont joué un rôle, comme nous l'avons déjà signalé. D'autre part, les critiques et les chercheurs dans le domaine littéraire ont tendance à donner beaucoup de crédit aux dires des écrivains. Cependant, tout chercheur en génétique sait que certaines affirmations des créateurs ne résistent pas à la confrontation avec les traces que leur processus créatif a laissées dans leurs documents de travail. Il ne s'agit pas évidemment de balayer ces témoignages, qui sont une source précieuse d'information, mais on ne peut pas non plus élaborer des théories sur la poétique et l'usage des langues chez un écrivain - qu'il soit monolingue ou plurilingue -, en se fondant uniquement sur des entretiens et des faits biographiques connus. Dans la mesure où l'on sait que les politiques éditoriales ne sont pas toujours favorables aux publications plurilingues, l'étude du texte, de la matérialité textuelle, voire avant-textuelle, permet d'accéder au monde créatif des écrivains habités par plusieurs langues, un monde trop souvent occulté par l'édition.

Les recherches entreprises dans le domaine du plurilinguisme littéraire montrent clairement que la vision monolingue de l'écrivain, de la littérature, de l'écriture est absolument infondée et qu'elle peut être remise en cause, notamment grâce à l'étude des documents de travail des écrivains ${ }^{29}$. Même dans le cas d'écrivains du XIXe siècle considérés comme fondateurs des littératures nationales modernes (Heine, Solomos, Tolstoï, Manzoni, Pouchkine...), on peut distinguer dans leur production en langue «nationale» l'influence d'autres langues et d'autres cultures, que l'on retrouve dans l'ensemble de leur production écrite.

Si ces faits sont bien connus des spécialistes, cela ne donne pas lieu forcément à des analyses approfondies du rôle des langues dans la création de ces écrivains et, surtout, ils sont généralement ignorés par le grand public.

Pour mieux comprendre la spécificité du plurilinguisme littéraire et des questions qui lui sont inhérentes, il faut maintenant revenir brièvement sur les premières manifestations de l'intérêt scientifique pour ce sujet ${ }^{30}$.

L'étude du plurilinguisme naît dans le domaine de la linguistique avant de s'étendre à celui de la littérature. En effet, les progrès de la linguistique ont permis de mieux cerner le fonctionnement du plurilinguisme en général, grâce à l'ouvrage Languages in Contact d'Uriel Weinreich (1952) ${ }^{31}$. Cet essai est issu des travaux de l'école linguistique de New York (par ailleurs fondamentale dans l'histoire du structuralisme) ${ }^{32}$. Uriel Weinreich y montre que, loin de constituer des univers fermés et autonomes, les langues entrent en contact dès qu'elles coexistent chez un locuteur et provoquent des phénomènes d'interférences. Weinreich distingue plusieurs types d'interférences, comme le changement de langue et le mélange de langues. Tous les niveaux de la langue sont affectés : phonétique, lexique et syntaxe. Mais

29. En France, l'étude des manuscrits des écrivains plurilingues a été initiée par les recherches menées en 2005-2007 dans le cadre du programme franco-russe Multilinguisme et genèse de textes (Académie des sciences de Russie/ CNRS), qui a conduit à la formation d'une structure institutionnelle - l'équipe de recherche « Multilinguisme, traduction, création » - au sein de l'ITEM.

30. Cet état de la recherche est exposé en détail dans Emilio Sciarrino, Le plurilinguisme en littérature. Le cas italien, Paris, Éditions des archives contemporaines, coll. «Multilinguisme, traduction, création », 2016, p. 13-22. 31. Uriel Weinreich, Languages in Contact. Findings and Problems, New York, Linguistic Circle of New York, 1953. 32. Concernant l'importance de l'école de linguistique de New York dans l'histoire du structuralisme, voir François Dosse, Histoire du structuralisme. Tome I : le champ du signe, 1945-1966, Paris, Éditions La Découverte, 2012, p. 77. 
les interférences ne dépendent pas seulement de la nature des langues. Le contact entre les langues peut également être influencé par des facteurs externes (comme la compétence linguistique d'un locuteur ou bien sa situation sociale).

C'est quelques décennies plus tard que la recherche s'intéressera véritablement au plurilinguisme littéraire, notamment après la publication de l'essai de Mikhaïl Bakhtine Problèmes de la poétique de Dostö̈evski (traduit en français en 1970) ${ }^{33}$ qui introduit la notion de polyphonie. Les approfondissements du concept de plurilinguisme à partir des années 1980 surviennent pendant le déclin de la pensée structuraliste, que les lectures françaises de Bakhtine ont contribué à sceller. Ces approches considèrent le plurilinguisme comme un concept culturel au sens large, ayant des enjeux philosophiques et politiques. Il s'agira alors de remettre en question le monolinguisme, en montrant que la langue unique se diffracte et se démultiplie toujours de façon ambivalente, qu'elle se compose d'un ensemble de forces et de devenirs. Cette interprétation, défendue notamment par Gilles Deleuze et Félix Guattari, se réfère à de nombreux écrivains plurilingues : Franz Kafka, Samuel Beckett, Ghérasim Luca, Pier Paolo Pasolini ${ }^{34}$. Ces auteurs appartiendraient tous à une «littérature mineure», notion que les deux philosophes reprennent à Kafka et qu'ils chargent de significations politiques 35 .

Quelques années plus tard, Jacques Derrida donne à son tour au plurilinguisme toute son importance philosophique. Partant d'une déconstruction du «logos », c'est-à-dire d'une critique de la raison occidentale centrée sur la parole écrite, le philosophe trouve dans le plurilinguisme un exemple idéal de l'impossibilité de réduire le langage à l'expression d'une signification univoque. Si ses études sur Paul Celan ${ }^{36}$ et sur James Joyce ${ }^{37}$ deviennent des références, c'est surtout dans Le Monolinguisme de l'autre 38 qu'il conceptualise l'opposition entre plurilinguisme et monolinguisme, inspirée notamment par Édouard Glissant et par Abdelkébir Khatibi39. En somme, ce moment culturel s'éloigne de l'approche formelle et réaffirme l'opposition entre monolinguisme et plurilinguisme, en insistant surtout sur ses enjeux éthiques et politiques.

Pour résumer, les études sur le plurilinguisme se sont éloignées de plus en plus de l'analyse linguistique du texte pour tendre vers une réflexion théorique, au risque de perdre de vue la singularité irréductible de chaque écriture. La limite d'une telle approche, dès lors, est de diluer le plurilinguisme (réel ou supposé) du texte dans un plus vaste univers conceptuel, tout en valorisant quelques exemples canoniques qui sont censés représenter l'univers entier des écrivains plurilingues. De plus, à la scission entre forme et contenu s'ajoute la confusion entre l'œuvre littéraire elle-même et le discours théorique qui l'environne 40 . À l'inverse, en remettant le texte et l'avant-texte au cœur des recherches sur le plurilinguisme littéraire,

33. Mikhaïl Bakhtine, Problèmes de la poétique de Dostoïevski [1929], traduit par Guy Verret, Lausanne, L'Âge d'homme, 1970 .

34. Gilles Deleuze, Carmelo Bene, Superpositions, Paris, Les Éditions de Minuit, 1979, p. 107.

35. Gilles Deleuze, Felix Guattari, Kafka. Pour une littérature mineure, Paris, Les Éditions de Minuit, 1975.

36. Jacques Derrida, Schibboleth : pour Paul Celan, Paris, Galilée, 1986.

37. Jacques Derrida, Ulysse gramophone, Paris, Galilée, 1987.

38. Jacques Derrida, Le Monolinguisme de l'autre, Paris, Galilée, 1996.

39. Ces deux auteurs sont cités en épigraphe dans Jacques Derrida, Le Monolinguisme de l'autre, op. cit.

40. François Cusset, French Theory. Foucault, Derrida, Deleuze \& Cie et les mutations de la vie intellectuelle aux

États-Unis, Paris, Éditions La Découverte, 2003, p. 232-240. 


\section{Définir la situation plurilingue d'un écrivain grâce à ses archives}

la critique génétique permet de définir précisément la situation plurilingue de chaque auteur et d'identifier la réalité des processus d'écriture plurilingue.

L'étude des archives permet d'accéder à la réalité des situations plurilingues des auteurs sans tomber dans les clichés (qui sont parfois entretenus par les écrivains eux-mêmes). Pour connaître et étudier véritablement un écrivain plurilingue, il faut, en effet, comprendre sa situation dans la maîtrise et l'usage des langues en cernant ses compétences linguistiques réelles pour saisir comment elles sont mises en œuvre dans son écriture. Dans ce but, loin de se satisfaire d'hypothèses ou d'idées reçues, il est nécessaire d'identifier les archives disponibles d'un écrivain plurilingue (ici le chercheur est parfois confronté à la relative difficulté d'accéder aux documents qui peuvent être dispersés dans plusieurs pays) afin d'explorer les traces de plurilinguisme dans ses documents de travail, dans sa correspondance, etc.

Par exemple, une bibliothèque d'auteur plurilingue constitue un terrain particulièrement intéressant, car elle peut révéler l'ensemble des langues sollicitées par l'écrivain dans ses lectures quotidiennes, ainsi que la proportion de chacun de ces idiomes, en d'autres termes son environnement linguistique et intertextuel le plus intime ${ }^{41}$.

Les correspondances - où souvent les langues mobilisées dépendent aussi des destinataires - regorgent également d'informations sur le contexte familial de l'écrivain, sur les détails de son parcours transnational (itinéraires des voyages, lectures et influences artistiques, environnement professionnel...) et, quelquefois, sur son processus créatif. Les archives épistolaires témoignent ainsi du lien vital que l'auteur entretient avec une plus vaste communauté déterritorialisée. Comme le montrent les archives de Giuseppe Ungaretti évoquées dans ce numéro par Emilio Sciarrino, les correspondances avec les traducteurs sont souvent riches en informations sur la genèse de l'œuvre plurilingue, tout en montrant le lien étroit qui existe entre traduction et création.

Quand ils sont disponibles, des documents tels que cahiers, journaux personnels et autres écrits autobiographiques demeurent bien entendu essentiels pour documenter en détail la trajectoire d'un écrivain. Dans les cas des écrivains peu connus ou oubliés, ces documents deviennent même indispensables, car comment connaître autrement la biographie d'un auteur jamais étudié auparavant? Correspondances et journaux personnels, où l'on rencontre habituellement de nombreux phénomènes plurilingues, permettent également de déterminer s'il existe une continuité entre l'univers linguistique qui environne l'écrivain et le monde de sa création.

Cela nous conduit aux brouillons et aux documents préparatoires de l'œuvre, qui sont, bien sûr, dépositaires d'informations cruciales quant aux rôles des langues dans le processus d'écriture. Ces documents témoignent de la richesse foisonnante des langues mobilisées pendant la rédaction par les écrivains polyglottes (voire hyperpolyglottes ${ }^{42}$ ), impliquant,

41. Le cas échéant, il est possible de mettre au jour des marginalia comme des listes de vocabulaire, des traductions ou des réflexions métalinguistiques, en somme les traces d'un apprentissage linguistique. C'est par exemple le cas de la bibliothèque privée d'Amelia Rosselli, riche en informations sur la situation linguistique de l'auteure. À ce sujet, voir Emilio Sciarrino, Le plurilinguisme en littérature, op. cit., p. 112-113.

42. On définit comme hyperpolyglotte une personne qui parle plus de six langues (ce terme serait forgé par Richard Hudson en 2003. À ce sujet, voir Kenneth Hyltenstam (éd.), Advanced Proficiency and Exceptional Ability in Second Languages, Kenneth Hyltenstam, <https://www.researchgate.net/publication/306359940_The_polyglot_-_an_initial_ characterization_on_the_basis_of_multiple_anecdotal_accounts > (consulté en décembre 2017), Boston, Gruyter 
outre de nombreuses langues vivantes, des langues anciennes, des dialectes et même des langues inventées. Si ces documents permettent généralement d'identifier différentes stratégies d'écriture que nous détaillerons plus loin, dans les cas les plus éclatants la mise au jour d'inédits va jusqu'à dévoiler tout un pan de l'œuvre écrit dans une autre langue, comme pour Pessoa, le poète portugais aux nombreux hétéronymes ${ }^{43}$, dont certains composaient exclusivement en français et en anglais ${ }^{44}$.

L'étude scrupuleuse de ces archives plurilingues contribue ainsi à offrir un profil linguistique et historique de l'auteur beaucoup plus fin que celui qui se dégage uniquement d'après la lecture de son œuvre publiée et d'après les sources critiques, qui tombent souvent dans le préjugé monolingue ou bien, inversement, qui cèdent à la tentation de faire de tout auteur l'équivalent de James Joyce. Précisons qu'il ne s'agira pas seulement de jauger la compétence linguistique d'un auteur, puisqu'il existe une distance entre cette compétence et la performance écrite (en d'autres termes, l'usage littéraire d'une langue) ${ }^{45}$, mais surtout de comprendre comment l'écrivain emploie, voire met en scène, ses différents idiomes en fonction de multiples critères : par rapport à la posture qu'il souhaite adopter, en lien avec son univers esthétique et, bien sûr, en fonction de son projet poétique personnel.

Ce type d'approche permet de souligner plusieurs situations récurrentes chez les écrivains plurilingues. La plus manifeste est celle du bilinguisme ou de la diglossie, qui a significativement attiré l'attention des chercheurs ${ }^{46}$. Cependant, il faut observer qu'un duo de langues cache souvent une multiplicité linguistique bien plus grande à l'œuvre, ce que les premières études en la matière n'ont pas toujours souligné. Vladimir Nabokov était un véritable auteur trilingue et non bilingue ${ }^{47}$; Samuel Beckett, tout en partageant son œuvre entre le français et l'anglais, était aussi un fin connaisseur de l'italien et de l'allemand 48 . Autrement dit, il existe un continuum entre le bilinguisme et le plurilinguisme radical qui est la signature de certains

Mouton, 2016, p. 215, note 1). Les écrivains hyperpolyglottes sont nombreux : Tolstoï qui maîtrisait une quinzaine de langues, João Guimarães Rosa qui a appris une vingtaine de langues...

43. Cf. infra.

44. Fernando Pessoa, Poèmes en français, éd. Patricio Ferrari, Paris, La Différence, 2014.

45. Sur la distinction entre compétence et performance dans les cas de plurilinguisme voir les observations d'Aneta Pavlenko, Emotions and Multilingualism, op. cit., p. 6.

46. Michael Oustinoff, Bilinguisme d'écriture et autotraduction, Julien Green, Samuel Beckett, Vladimir Nabokov, op. cit.

47. Olga Anokhina, «Le rôle du multilinguisme dans l'activité créative de Vladimir Nabokov», dans Olga Anokhina (dir.), Multilinguisme et créativité littéraire, op. cit., p. 15-25; Olga Anokhina, «Vladimir Nabokov, un écrivain plurilingue », dans Évelyne Argaud, Malek Al-Zaum, Elena da Silva Akborisova (dir.), Le proche et le lointain : enseigner, apprendre et partager des cultures étrangères, Paris, Éditions des archives contemporaines, 2017, p. 1-7; Olga Anokhina, «Vladimir Nabokov et la langue française», dans Olga Anokhina et Alain Ausoni (dir.), Vivre entre les langues, écrire en français, Paris, Éditions des archives contemporaines, 2018 (à paraître).

48. À propos des références italiennes de Beckett, Dante en particulier, voir par exemple Daniela Caseli, Beckett's Dantes. Intertextuality in the Fiction and Criticism, Manchester, Manchester University Press, 2009. Quant à l'importance de l'allemand pour Beckett, voir Therese Fischer-Seidel et Marion Fries-Dieckmann (dir.), Der unbekannte Beckett : Samuel Beckett und die deutsche Kultur, Frankfurt-Main, Suhrkamp, 2005 ; Marion FriesDieckmann, Samuel Beckett und die deutsche Sprache. Eine Untersuchung der deutschen Übersetzungen des dramatischen Werks, Trier, Wissenschaftlicher Verlag Trier, 2007; Dirk Weissmann, " "Writing from the midzone" : réflexions sur la territorialité de l'écrivain plurilingue auto-traducteur», dans Ronald Jenn et Corinne Oster (dir.), Territoires de la traduction, Arras, Artois Presses Université, 2014, p. 95-106. 


\section{Vers une typologie des stratégies créatives plurilingues}

auteurs comme Joyce ou, dans une moindre mesure, Edoardo Sanguineti (qui employait dans ses poèmes l'italien, le français, l'anglais, l'allemand, l'espagnol, le latin et le grec ancien ${ }^{49}$.

Enfin, une autre de ces situations est le faux monolinguisme ou monolinguisme apparent. Celle-ci concerne les auteurs que l'on considère traditionnellement comme monolingues, mais dont le processus d'écriture a en fait mobilisé plusieurs langues. Pour ne citer qu'un exemple, le roman I Promessi Sposi d'Alessandro Manzoni, considéré comme le texte fondateur de la langue moderne italienne, a connu plusieurs phases de rédaction où le dialecte milanais était mélangé à du français, avant de connaître une nouvelle version normalisée selon la norme toscane 50 .

En plus de contribuer de façon cruciale à déterminer avec précision le profil linguistique de l'écrivain, la critique génétique permet d'identifier les stratégies récurrentes chez des écrivains qui appartiennent pourtant à des époques et à des aires culturelles très différentes.

L'observation des manuscrits de plusieurs écrivains plurilingues a ainsi mis en évidence, à partir des pratiques individuelles, quelques tendances générales dans l'usage des langues par ces écrivains singuliers. Nous avons pu présenter ailleurs une esquisse de cette typologie 51. Il nous semble néanmoins important de la détailler au sein de ce volume destiné aux généticiens en y ajoutant de nouveaux exemples et en l'ouvrant vers d'autres pratiques créatives utilisées par des écrivains plurilingues. Ces considérations théoriques ayant une portée générale ont été élaborées grâce à des travaux individuels, dont certains résultats sont diffusés dans des ouvrages récents 52 .

Quatre grandes stratégies, dont un certain nombre sera illustré par les contributions de ce numéro, ont été identifiées à ce jour : 1) la séparation fonctionnelle des langues, 2) le mélange des langues, 3) l'écriture parallèle en deux langues et 4) l'écriture consécutive ou l'autotraduction - mais il ne s'agit évidemment pas d'une liste exhaustive, chaque écrivain pouvant recourir à une palette variée de ces pratiques selon la phase créative, selon l'œuvre ou encore selon le moment de sa vie, même si des tendances générales sont bien identifiables pour chaque créateur.

\section{Séparation fonctionnelle des langues}

Les études des documents préparatoires de certains écrivains plurilingues ont pu montrer qu'ils utilisent deux langues lors de leur processus créatif mais qu'ils confèrent à chacune d'elles une tâche spécifique. La plupart du temps, l'une des langues est vouée à l'accompagnement et à la stimulation métalinguistiques du déploiement textuel dans une autre langue. Ainsi, chez un écrivain plurilingue qui utilise cette stratégie scripturaire, les commentaires métadiscursifs qui construisent une sorte de dialogue intérieur de l'écrivain avec lui-même - à l'instar des célèbres métaréflexions de Zola - sont rédigés dans une langue

\footnotetext{
49. Edoardo Sanguineti, Segnalibro. Poesie (1951-1981), Milano, Feltrinelli, 2010.

50. L'ensemble de la littérature italienne pourrait être lue à travers le prisme du monolinguisme et du plurilinguisme, d'après les analyses de Gianfranco Contini, Varianti e altra linguistica, Torino, Einaudi, 1970.

51. Olga Anokhina, «Étudier les écrivains plurilingues grâce aux manuscrits », dans Olga Anokhina et François Rastier (dir.), Écrire en langues, op. cit., p. 31-43.

52. Olga Anokhina, Natalia Vélikanova et al. (dir.), Multilinguisme et genèse des textes, op. cit., 2010 (en russe) et Olga Anokhina (dir.), Multilinguisme et créativité littéraire, op. cit.
} 
différente de celle choisie pour l'écriture du texte à proprement parler. Ce procédé créatif, qui accorde à chacune des langues de l'écrivain un rôle spécifique, a été utilisé par des écrivains plurilingues à différentes époques.

Le cas le plus célèbre est sans doute Pétrarque (XIVe), qui a été pourtant considéré, contrairement à Dante, comme un écrivain «monolingue 53 », et qui utilisait de fait le latin et l'italien lors de son processus de création, en accordant à chacune de ces langues un rôle bien défini. Il se donnait des indications métadiscursives en latin alors qu'il composait des vers en italien, comme l'a montré Maria Teresa Giaveri :

[...] les autographes du Chansonnier sont en effet parsemés de marques d'approbation ou d'insatisfaction par rapport au texte italien, inscrites en latin, telles que vel (pour introduire des variantes ponctuelles), hic placet, hoc placet, hec uidetur proximior perfectioni, ou bien non videtur satis triste principium, dic aliter hic, quand le vers n'est pas satisfaisant 54 .

Quelques siècles plus tard, on observe un phénomène très similaire dans les manuscrits d'Alexandre Pouchkine (XIXe). Boris Tomachevski55 affirme que, chez le poète russe, le français est sollicité surtout à l'étape de planification, alors que la langue russe surgit dès les premières tentatives de textualisation 56 . Tout comme chez Pétrarque, la répartition des langues chez Pouchkine se fait donc en fonction de l'étape ou de la phase créative 57.

On observe la même logique créative dans les manuscrits de l'écrivain Dionysios Solomos (1798-1857) présenté dans ce numéro par Kostis Pavlou. Le grand poète et fondateur de la littérature nationale grecque, dont la langue principale était l'italien, préparait une matrice de ses œuvres en prose, en langue italienne, et composait ensuite les vers en grec 58 .

Cependant, de ce processus créatif complexe s'appuyant sur plusieurs langues rien ne reste dans l'état publié de l'œuvre qui présentera, par conséquent, une apparence totalement monolingue.

\section{Code-switching ou le mélange des langues}

Contrairement aux écrivains qui pratiquent la séparation fonctionnelle des langues lors d'un processus créatif et qui produisent - du fait de cette séparation - une œuvre d'apparence

53. Contini oppose en effet Dante, écrivain plurilingue par excellence et Pétrarque, qui serait selon lui un écrivain «monolingue».

54. Maria Teresa Giaveri «Entre le latin et l'italien, entre la philologie et la génétique : le Manuscrit Vatican Latin 3196 de Pétrarque», dans Olga Anokhina (dir.), Multilinguisme et créativité littéraire, op. cit., p. 41-54. Ici et plus loin l'italique est utilisé pour différencier graphiquement deux langues présentes au sein du même texte.

55. Olga Anokhina, «Textología rusa y contribución teórica de Boris Tomashevski » [La textologie russe et l'apport théorique de Boris Tomachevski], dans A. Arcocha-Scarcia, J. Lluch-Prats et M. J. Olaziregi (dir.), En el taller del escritor: Génesis textual y edición de textos, San-Sebastian, Éditions de l'université du Pays Basque, 2010, p. 71-92 (en espagnol).

56. Boris Tomachevski, L'Écrivain et le Livre, Moscou-Leningrad, Priboï, 1928 (en russe).

57. Sur les étapes de travail créatif, dans le domaine de la génétique voir Pierre-Marc de Biasi, Génétique des textes, Paris, Nathan, 2011 et dans le domaine de la psychologie cognitive John R. Hayes et Linda S. Flower, «Identifying the organization of writing processes», dans Lee W. Gregg \& Erwin R. Steinberg (dir.), Cognitive processes in writing, Hillsdale (NJ), Lawrence Erlbaum Associates, 1980, p. 3-30.

58. Pour une analyse détaillée, voir Kostis Pavlou, La Genèse du premier projet d'écriture des Libres Assiégés de Dionysios Solomos : une approche génétique, thèse de doctorat, université de Paris IV, 2012. 
monolingue, les écrivains qui ont recours au code-switching se manifestant par le mélange des langues dans leurs écrits sont les seuls qui donnent à voir l'importance des langues pour leur création, y compris dans les textes publiés, même si leur réalité créative plurilingue est beaucoup plus importante lors de leur processus d'écriture (manifeste dans leurs documents de travail) que dans leurs œuvres publiées.

Ces écrivains utilisent donc une stratégie créative diamétralement opposée à la séparation fonctionnelle des langues. Le code-switching a été étudié et théorisé dans les années 1970 par la sociolinguistique, qui a observé la production orale des locuteurs plurilingues. Or, ce mélange de deux ou plusieurs codes linguistiques, fréquent chez des locuteurs plurilingues, est également très présent dans les brouillons des écrivains plurilingues, dans leurs échanges épistolaires et même dans leurs œuvres publiées.

Le code-switching ostentatoire caractérise notamment la production de certains écrivains américains d'origine hispanique ${ }^{59}$ où le mélange des deux langues, l'espagnol et l'anglais ${ }^{60}$, est très présent dans les œuvres publiées 61 :

Estoy sentada en el comedor de nuestro departamento, taipeando en el laptop de Pierre which somehow, de milagro, did NOT get ruined by the sheets of water que inundaron nuestro departamento while we were in Chile. Si hay un Dios, his/her name is écriture, porque although we returned to the disconcerting fact of una cañería revantada in the departamento above our, y muchísimo water damage in our apartment, the computer, my work and most of my books were spared (my first editions of Pizarnik, unscathed!) ${ }^{62}$.

En ce qui concerne les écrivains européens, même si leurs œuvres publiées sont davantage conformes aux normes linguistiques unilingues que celle des écrivains chicanos, en réalité, au cours de leur activité d'écriture ils sont aussi très nombreux à mélanger les codes linguistiques 63 .

Nous avons mentionné plus haut le fait que Dionysios Solomos essaye, en principe, de séparer l'écriture de vers en grec et la rédaction en prose en langue italienne, mais dans ses manuscrits on trouve également des passages où les deux langues se mélangent et leur relation devient plus complexe.

Chez Pouchkine, qui - comme nous l'avons vu - respecte habituellement la séparation fonctionnelle des langues lors de l'écriture d'une œuvre en prose, en réservant le français pour

59. Selon Raymund Paredes, «By 1900, Mexican American literature had emerged as a distinctive part of the literary culture of the United States». Elle s'est consolidée avec la publication en 1945 du roman de Josephina Niggli Mexican Village, qui fut «the first literary work by a Mexican American to reach a general American audience». Voir Raymund Paredes, «Teaching Chicano Literature: An Historical Approach », dans Heath Anthology Newsletter, $\mathrm{n}^{\circ}$ 12, 1995, <http://faculty.georgetown.edu/bassr/tamlit/essays/chicano.html > (consulté en décembre 2017).

60. Ilan Stavans, Spanglish: The Making of a New American Language, London, HarperCollins Publishers Inc., 2003. 61. À ce sujet, voir par exemple, Dominique Breton et Sabine Tinchant-Benrahho, «Traduire le spanglish ou la "fabrique du lecteur" en question", dans La Main de Thôt, $\mathrm{n}^{\circ}$ 2, 2014, disponible en ligne : <https://revues.univ-tlse2. fr/lamaindethot/index.php?id=270> (consulté en décembre 2017).

62. Susana Chávez-Silverman, Killer Crónicas: Bilingual Memories, Wisconsin, University of Wisconsin Press, 2004 : <https://uwpress.wisc.edu/index.html> (consulté en décembre 2017).

63. Un numéro récent de la revue électronique Continents Manuscrits (Revues.org) questionne précisément les limites de ce qu'on peut appeler la «norme» et le «linguistiquement correct». Cf. Olga Anokhina, Florence Davaille, Hervé Sanson (dir.), Bien écrire/mal écrire : écrire en "périphérie » de la norme (écrivains plurilingues, écrivains «francophones»), Continents manuscrits, n 2, 2014, <http://journals.openedition.org/coma/219>. 
la planification et le russe pour la textualisation, on peut cependant observer un phénomène de code-switching dans sa composition poétique et dans sa correspondance privée. L'alternance des codes chez Pouchkine a la plupart du temps une valeur ironique, humoristique comme dans ce poème :

А что театр? [Et le théâtre ?]

О! Сиротеет [oh, il est abandonné],

C'est bien mauvais, ça fait pitié.

Тальма совсем оглох, слабеет [Talma est devenu sourd et s'affaiblit],

И мамзель Марс [et Mlle Mars]

увы! стареет [hélas vieillit],

Зато Потье [mais Potier], le grand Potier!

Même dans certains plans de Pouchkine, on observe un mélange très prononcé de français et de russe :

История Федора Орлова. Un élégant, un Zavadovski, mauvais sujet, des maîtresses, des dettes. Он влюбляется в бедную светскую девушку, увозит ее; первые годы роскошные, впадает в бедность, cherche des distractions chez ses premières maîtresses, devient escroc et duelliste. Доходит до разбойничества, зарезывает Щепочкина; застреливается (или исчезает) ${ }^{64}$.

[Histoire de Fiodor Orlov. Un élégant, un Zavadovski, mauvais sujet, des maîtresses, des dettes. Il tombe amoureux d'une pauvre jeune fille, part avec elle; les premières années opulentes, devient pauvre, cherche des distractions chez ses premières maîtresses, devient escroc et duelliste. Se transforme en bandit, tue Schepotchkine; se suicide (ou disparait).]

Ce plan de Pouchkine montre que la distribution fonctionnelle des langues n'est pas toujours si nette chez l'écrivain russe que l'affirmait Boris Tomachevski. En réalité, chez Pouchkine, l'interaction des langues est différente pour chaque œuvre. Ainsi, les plans des œuvres non achevées, et surtout celles dont la textualisation n'a pas été entamée, sont systématiquement écrits en français, alors que pour son célèbre roman La Fille du capitaine, le français «n'est

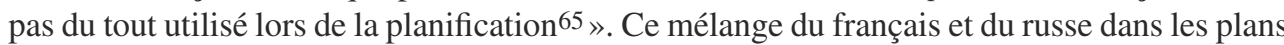
qui représente, à notre avis, une cristallisation du langage intérieur ${ }^{66}$, nous amène à penser que les deux langues forment chez Pouchkine - et chez bien d'autres écrivains plurilingues (voir par exemple la contribution de Delfina Cabrera sur Manuel Puig ici même) - une matrice linguistique commune dans laquelle sa pensée puise indifféremment 67 .

Chez les écrivains qui pratiquent le mélange des codes linguistiques, il est rare qu'une des langues maîtrisées domine absolument les autres, chacune d'elles apportant plutôt une contribution - plus ou moins facilement identifiable - au processus créatif, plurilingue par

64. Alexandre S. Pouchkine, Euvres complètes en 10 volumes, Moscou, Académie des sciences de Russie, 1956-1958, t. VI, p. 797. Le plan du roman «Русский Пелам» [Pélame russe].

65. Ekatérina Dmitirieva, «O некоторых вопросах билингвизма пушкинского письма и пушкинского эпистолярия » [Certains aspects du bilinguisme de l'écriture créative et de la correspondance chez Pouchkine], dans Olga Anokhina, Natalia Velikanova et al. (dir.), Multilinguisme et genèse du texte, op. cit., p. 62-81, ici p. 64. 66. Olga Anokhina, «The Genesis of Texts: Planning and Interior Language », Belgian Journal of Linguistics, $\mathrm{n}^{\circ} 23$, «New Approaches in Textual Linguistics», 2009, p. 63-72.

67. Il semblerait donc que lors de l'étape de planification, la pensée et le langage intérieur de l'écrivain puisent indifféremment dans les deux langues, alors que la phase de la textualisation impose une séparation nette des deux langues du poète. 
essence. L'importance de toutes ces langues est si forte pour ces créateurs qu'ils cherchent à les manifester dans leurs œuvres publiées, même si, du point de vue quantitatif, la présence des éléments hétérolingues est souvent moins importante dans une œuvre publiée par rapport à ce qu'on peut déceler dans ses dossiers préparatoires.

Le lecteur du seul texte publié est donc confronté à la question fondamentale qui se pose d'un point de vue génétique : pour quelle raison, choix délibéré ou compulsion, telle langue paraît-elle préférable à telle autre en un point déterminé ? On ne peut guère espérer résoudre cette question sans avoir recours aux brouillons. On peut par exemple penser à Léon Tolstoï dont le célèbre roman Guerre et paix contient des pages entières en français au sein même du texte russe publié68. Or, dans les manuscrits de ce grand écrivain russe, le français est présent partout : dans les plans, dans les notes, dans les carnets, dans les scénarios, dans les brouillons ainsi que dans la marge des ouvrages de sa bibliothèque personnelle. Tolstoï utilise une sorte de langue mixte franco-russe qu'on pourrait qualifier d'idiolecte tolstoïen, non seulement à l'étape de planification, mais également aux étapes assez avancées de la textualisation. On observe de nombreuses interférences provenant du français à différents niveaux :

- graphique (Nicolas и Nаташа в деревне [Nicolas et Natacha à la campagne]);

- lexico-morphologique («вел жизнь парижского viveur в Петербурге» [il menait la vie d'un parisien viveur à Petersbourg]; «Вот настоящая, дорогая chair à canon» [Voici une véritable [et] coûteuse chair à canon]; «танцовали в зале шестую anglaise» [on dansait dans la salle une sixième anglaise $]$ );

- syntaxique («для меня это все еще un mauvais drôle, om которого долго не будет людям покоя» [pour moi il reste un mauvais drôle, qui ne laissera personne en paix encore bien longtemps]);

- phraséologique («Idée fixe - становится отдать замуж дочерей» [Son idée fixe devient marier ses filles]; «Поэзия comme il faut или грубого разврата» [La poésie du comme il faut ou d'une profonde déchéance]; «Rattraper le temps perdu старик волочится за всеми» [Rattraper le temps perdu le vieillard courait après tous les jupons]; «Главное faire de l'argent » [l'essentiel c'est de faire de l'argent] $)^{69}$.

Certaines de ces notes franco-russes arrivent jusqu'à la phase pré-éditoriale, voire éditoriale. De son vivant, l'écrivain s'attelait alors à «nettoyer» ses textes d'une omniprésence du français, relayé dans ce «combat» par d'autres personnes participant activement à l'édition de ses textes, comme son épouse ou son secrétaire. Ce combat se poursuivra dans toutes les éditions ultérieures de ses romans, chaque nouvelle édition ayant pour objectif de «russiser» encore davantage le texte original hétérolingue de Tolstoï. Le cas illustre le véritable décalage qui existe entre le processus créatif fortement plurilingue et la pression éditoriale qui tend souvent vers le monolinguisme.

Plus près de nous, Vladimir Nabokov lui aussi puisait constamment dans une matrice plurilingue composée des langues russe, française et anglaise de sorte que les interférences

68. Olga Anokhina, «Écrivains russes du XIXe siècle : écrivains plurilingues ? », dans Olga Anokhina, Till Dembeck and Dirk Weissmann (dir.), Mapping Multilingualism in 19th Century European Literatures - Le plurilinguisme dans les littératures européennes du XIXe siècle, LIT-Verlag, 2018 (à paraître).

69. Nous remercions chaleureusement Natalia Vélikanova qui nous a fourni ces exemples. 
linguistiques se rencontrent fréquemment dans l'ensemble de sa production : dans les brouillons, dans les lettres et dans les œuvres publiées. Quand on observe de près les néologismes et les jeux de mots omniprésents dans ses œuvres anglophones, on constate que, bien souvent, cette créativité linguistique est motivée par les langues russe et française ${ }^{70}$.

À l'inverse, l'exemple qui suit montre clairement la prégnance de la langue française lors de l'écriture en russe. Il provient de son dernier roman écrit en russe, le Don (1937), considéré comme le sommet de la création de Nabokov en langue russe et l'un des chefs-d'œuvre de la littérature russe du $\mathrm{XX}^{\mathrm{e}}$ siècle. Il montre à quel point la connaissance de la langue française modèle son écriture en russe 71 :

Все равно. J'aime l'épée qui brille, le poisson qui frétille et le petit ventre de ma gentille. (Откуда? Сережа Боткин любил повторять.)

«В среду, там же». Oui, si tu veux, ça me va... Но ты наверное придешь? Она ответила, что никогда не подкладывает никаких кроликов.

[Peu importe. J'aime l'épée qui brille, le poisson qui frétille et le petit ventre de ma gentille. (D'où ça vient? Sereja Botkine aimait à le répéter.)

«Mercredi, au même endroit». Oui, si tu veux, ça me va... Mais es-tu sûre de venir? Elle a répondu qu'elle ne pose jamais de lapins. $]^{72}$

Dans cet exemple, on voit comment une chansonnette (J'aime l'épée qui brille, le poisson qui frétille et le petit ventre de ma gentille), un bout de dialogue (Oui, si tu veux, ça me va) et une expression idiomatique (poser un lapin) en langue française s'intègrent naturellement dans le texte russe. Notons que cet exemple est particulièrement parlant, l'expression poser un lapin n'ayant aucun sens pour le locuteur monolingue russe, mais elle appartient à la matrice linguistique de l'écrivain et apparaît, de ce fait, dans l'un des états rédactionnels de son roman.

L'étude des manuscrits et de la correspondance de Nabokov permet de voir que son recours à d'autres langues au sein de ses écrits n'est pas un simple procédé stylistique ${ }^{73}$. Il s'agit de la réalité linguistique et cognitive d'un locuteur plurilingue qui passe naturellement d'une langue à une autre. Il maîtrise parfaitement les différents codes linguistiques mais ne peut pas rester enfermé dans le cadre étroit d'une seule langue. Le plurilinguisme et le multiculturalisme inhérents à cette personne trouvent alors leur expression soit à l'oral dans des situations appropriées, soit à l'écrit, comme c'est le cas pour les écrivains plurilingues.

L'exemple de Pessoa présenté dans ce numéro par João Dionísio nous a permis d'identifier une autre stratégie, celle du changement de langue définitif au cours de la genèse : au cours de l'écriture, l'écrivain abandonne une langue pour passer - définitivement - à une autre. Il s'agit

70. Jane Grayson, Nabokov translated. A comparison of Nabokov's Russian and English Prose, Oxford, Oxford University Press, 1977.

71. Ce document manuscrit, qui contient des éléments qui n'ont jamais été intégrés dans l'état publié de l'œuvre, est décrit et analysé en détail par Jane Grayson dans Jane Grayson, Метаморфозы «Дара» [Métamorphoses du Don], dans Владимир Набоков: pro et contra [Vladimir Nabokov : pro et contra]. En ligne : <http://coollib. com/b/156848/read\#t89>.

72. Notre traduction. Library of Congress, Manuscript Division, Vladimir Nabokov, Dar, Box 2, Folder 6 (cahier), n.d. dernière page.

73. Olga Anokhina, «Vladimir Nabokov : du style et des langues», dans Stéphane Bikialo et Sabine Pétillon (dir.), La Licorne, numéro spécial «Dans l'atelier du style. Du manuscrit à l'œuvre publiée », 2012, n 98, Rennes, Presses universitaires de Rennes, p. 211-220. 
d'une sorte de «genèse avortée » quand l'écrivain n'arrive pas à avancer. Pessoa commence à écrire un poème en français ou en anglais mais passe très rapidement au portugais qui lui permet d'exprimer plus pleinement sa créativité. À la différence des écrivains chicanos ou de Nabokov chez qui le code-switching est un phénomène permanent, chez Pessoa le changement de langue intervient au début du processus créatif.

Il existe deux autres stratégies créatives (écriture simultanée en deux langues et écriture consécutive en deux langues) qui donnent lieu à deux œuvres monolingues parfaitement distinctes, écrites en deux langues différentes. Par conséquent, ces deux œuvres sont destinées aux lecteurs de langue et de nationalité différentes et ont chacune leurs propres réceptions sur les marchés éditoriaux des deux pays. Quelquefois, l'existence d'une œuvre jumelle dans une autre langue de l'écrivain n'est même pas mentionnée et se trouve par conséquent ignorée par le public et par la critique. Cependant, comme nous allons le voir à présent, le processus créatif de ces œuvres en apparence séparées et indépendantes fait partie d'un seul et même processus dont on peut avoir un aperçu uniquement grâce aux documents de travail de l'écrivain.

\section{Écriture simultanée en deux langues}

L'écriture simultanée ou parallèle en deux langues est une stratégie créative assez rare, employée surtout dans le cas de la composition poétique bilingue. En effet, de nombreux poètes ont donné naissance à des poèmes jumeaux 74 en au moins deux langues. Ces poèmes doubles peuvent être intégrés dans une seule œuvre publiée qui aura une identité plurilingue manifeste, voire revendiquée (poètes chicanos, Desbiens, Federman), ou bien ils peuvent aussi donner lieu à deux œuvres séparées qui auront une apparence monolingue (Celan, Rabearivelo). Dans ces deux cas, il est impossible de savoir comment ont été créées ces œuvres jumelles sans avoir accès aux traces du processus créatif qui a amené à leur émergence.

Cette stratégie créative singulière a pu être identifiée grâce aux recherches de Claire Riffard sur les manuscrits du poète malgache Jean-Joseph Rabearivelo (1903-1937) 75. L'observation de ses documents de travail, dont un échantillon est présenté dans ce numéro, a permis d'établir la véritable logique créative de certains de ses poèmes doubles. En effet, contrairement aux affirmations contradictoires de Rabearivelo sur la primauté d'écriture en l'une ou en l'autre de ses deux langues, français et malgache, les pages du cahier manuscrit divisées en deux parties laissent voir la relation très complexe de stimulation mutuelle entre les deux langues parlées par le poète.

Cette même stratégie créative est quelquefois utilisée par Manuel Puig, présenté dans ce volume par Delfina Cabrera. Même si l'analyse approfondie de la genèse de certaines de ses

74. Pour reprendre le terme «twin poems » de Federman dans Raymond Federman, A voice within a voice: Federman translating/translating Federman, 1996, <www.federman.com/rfsrcr2.htm > (consulté en décembre 2017).

75. Pour une analyse détaillée, voir Claire Riffard, «Étude des manuscrits malgaches bilingues de J. J. Rabearivelo», dans Olga Anokhina (dir.), Multilinguisme et créativité littéraire, op. cit., p. 55-65. On voit un fonctionnement similaire dans l'écriture d'autres poètes bilingues, comme par exemple chez Patrice Desbiens. À ce sujet, voir Mathieu Simard, «Norme unilingue/Norme multilingue : revisiter le bilinguisme littéraire de l'écrivain franco-ontarien Patrice Desbiens », dans Olga Anokhina, Florence Davaille, Hervé Sanson (dir.), Bien écrire/mal écrire : écrire en «périphérie» de la norme (écrivains plurilingues, écrivains «francophones»), op. cit. En ligne : <http://journals. openedition.org/coma/313 >. 
œuvres montre la simultanéité de la campagne de correction, son écriture de deux versions en espagnol et en anglais relève d'un processus consécutif qui s'approche de l'autotraduction, c'est-à-dire de la recréation par l'auteur du texte de cette même œuvre dans une autre langue. Généralement, cette recréation (ou traduction) est opérée par l'écrivain une fois que ce texte a déjà une forme plus ou moins finalisée dans une autre langue. Pour cette raison, nous appelons l'écriture consécutive en deux langues, dont il sera question plus loin, autotraduction.

Même si la notion de simultanéité a ses limites («En allant plus loin, on s’aperçoit toutefois que l'opposition entre successivité et simultanéité est plus superficielle qu'il n’y paraît. Il y a toujours de la successivité dans la cocréation simultanée ${ }^{7}$ »), elle est néanmoins essentielle pour distinguer et définir les stratégies créatives plurilingues.

\section{Autotraduction ou comment annuler la clôture du texte}

Contrairement à l'écriture en parallèle qui engendre pratiquement en même temps deux œuvres en deux langues différentes et qui semble assez minoritaire dans l'arsenal des stratégies scripturaires utilisées par des écrivains plurilingues, l'autotraduction qui opère de manière consécutive en deux (ou en plusieurs) langues est extrêmement répandue, et constitue même une pratique scripturaire majoritaire. En effet, un grand nombre d'écrivains plurilingues soit effectuent eux-mêmes les traductions de leurs œuvres (comme Nancy Huston, Samuel Beckett, Vassilis Alexakis, Anne Weber, et bien d'autres), soit supervisent les traductions faites par leurs collaborateurs (comme Vladimir Nabokov ou Günter Grass ${ }^{77}$ ). Face à l'opacité des indices paratextuels qui accompagnent la publication des textes autotraduits, c'est le recours aux documents de travail qui là aussi permet d'établir la part de chacun, écrivain et traducteur(s), dans le processus collaboratif de recréation d'une œuvre dans une autre langue. Les études génétiques, comme celle d'Ungaretti présentée dans ce volume par Emilio Sciarrino, offrent des informations essentielles quant à ces processus d'écritures hybrides qui combinent la traduction et la création littéraire.

Il y a de nombreuses raisons pour lesquelles les écrivains s'autotraduisent. Pour certains, s'investir soi-même dans la traduction de leur œuvre permet de pallier la défiance qu'ils ont envers leurs traducteurs. Pour d'autres, l'autotraduction permet de cerner les imperfections, les faiblesses du texte : elle sert alors d'une sorte de miroir au texte déjà écrit - phénomène brillamment illustré ici par l'étude d'Aurelia Arcocha-Scarcia qui porte sur le processus créatif plurilingue de Juan Mari Lekuona. Mais la véritable - ou, du moins, principale raison qui motive les écrivains plurilingues à s'autotraduire réside sans doute dans le fait que l'autotraduction leur permet de continuer à écrire, en annulant ainsi la clôture du texte ${ }^{78}$. Les chercheurs en génétique savent bien à quel point les écrivains résistent à cette clôture,

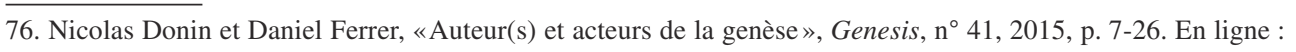
$<$ http://journals.openedition.org/genesis/1440>.

77. Günter Grass considérait ses traducteurs comme sa «grande famille». Il les réunissait régulièrement pour passer en revue les difficultés que recelaient ses textes. Céline Letawe, «Günter Grass and His Translators: From a Collaborative Dynamic to an Apparatus of Control? », dans Anthony Cordingley et Céline Frigau Manning (dir.), Collaborative translation: From the Renaissance to the digital age, London, Bloomsbury, 2016, p. 130-144.

78. Olga Anokhina, «L'autotraduction ou Comment annuler la clôture du texte?», dans Anna Lushenkova Foscolo et Malgorzata Smorag-Goldberg (dir.), Plurilinguisme et auto-traduction : langue perdue, langue sauvée, Paris, Institut d'études slaves, 2018 (sous presse). 
en repoussant encore et encore le moment inéluctable du «bon à tirer». Les écrivains plurilingues ont cette possibilité extraordinaire de déjouer la contrainte de la clôture, de dépasser l'achèvement d'une œuvre consolidée par son édition ${ }^{79}$ en la réécrivant dans une autre langue. À la différence de ceux qui produisent deux textes quasi identiques $(\mathrm{A}=\mathrm{B})$, comme le fait par exemple Manuel Puig, chez certains écrivains plurilingues, la traduction de leur texte vers une autre langue les amène à sa réécriture partielle, voire complète. À son tour, cette nouvelle version les fait revenir sur leur texte initial pour le modifier à nouveau en instaurant ainsi un processus créatif circulaire : $\mathrm{A} \rightarrow \mathrm{B} \rightarrow \mathrm{A}^{80}$. L'écriture en langue $\mathrm{B}$ fait donc partie à la fois de sa propre genèse mais aussi de la genèse de l'œuvre en langue A en constituant un état textuel intermédiaire qui joue le rôle d'une révision déguisée.

La porosité des frontières entre l'écriture plurilingue, la traduction effectuée en solitaire - l'autotraduction à proprement parler - et la traduction en collaboration avec l'écrivain, a engendré un grand nombre de travaux et de tentatives d'établir la typologie de ces pratiques. Les modalités intermédiaires ont été identifiées et désignées de différentes manières par des chercheurs : la semi-autotraduction ${ }^{81}$, l'autotraduction partagée 82 , (l'auto)traduction partiellement auctoriale 83 ou encore l'autotraduction indirecte ${ }^{84}$. Ces désignations soulignent la complexité des phénomènes autotraductifs et collaboratifs qui ne peuvent être mis au jour qu'à l'aide des documents de travail.

\section{Émergence d'une conscience métalinguistique}

Nous souhaiterions à présent attirer l'attention sur une caractéristique qui est propre aux écrivains de manière générale mais qui se trouve particulièrement renforcée chez des écrivains plurilingues. Il s'agit de la conscience métalinguistique ${ }^{85}$. Celle-ci peut être définie comme une «habileté à réfléchir sur le langage qui devient l'objet de notre pensée $86_{\text {». }}$.

Les recherches en psychologie cognitive et en psycholinguistique ont montré que le bilinguisme chez des enfants favorise le développement de leur conscience métalinguistique.

79. Jean-Louis Lebrave, «L'écriture inachevée», dans Dominique Budor et Denis Ferraris (dir.), Objets inachevés de l'écriture, Paris, Presses de la Sorbonne Nouvelle, 2001, p. 19-30.

80. Olga Anokhina, «Cas limites d'autotraduction : cercle, spirale, chaos», dans Esa Hartmann et Patrick Hersant (dir.), Au miroir de la traduction : avant-texte, intratexte, paratexte, Paris, Éditions des archives contemporaines, 2018 (sous presse).

81. Xosé Manuel Dasilva, «O dilema de traducir a obra autotraducida», dans International Conference Self-translation in the Iberian Peninsula, University College Cork, 20-21 septembre 2013 et Xosé Manuel Dasilva, «En torno al concepto de semiautotraducción», dans Quaderns, revista de traducció, n 23, 2016, p. 15-33.

82. Julio-César Santoyo, «Autotraducciones: ensayo de tipología», dans Pila Martino Alba, Juan Antonio Albaladejo Martínez, Marta Pulido (dir.), Al humanista, traductor y maestro Miguel Ángel Vega Cernuda, Madrid, Editorial Dykinson, 2012, p. 205-222 (citées ici : p. 216-217).

83. Maria Recuenco Penavlver, «Más allá de la traducción: la autotraducción », dans Trans. Revista de Traductología, 15, 2011, p. 193-208 (citée ici : p. 206).

84. Josep Miguel Ramis, Autotraducció i Sebastià Juan Arbó. El cas de «Terres de l'Ebre», Barcelona, Universitat Pompeu Fabra, Tesis de doctorado dirigida por Enric Gallén Miret, 2011 (citée ici : p. 153).

85. Olga Anokhina, «Conscience métalinguistique : de l'apprenant de langues à l'écrivain plurilingue », dans Olga Anokhina (dir.), Plurilinguisme, numéro spécial du Nouveau Bulletin de l'ADEAF, n 127, mars 2015, p. 18-20. 86. Pour les définitions de la conscience linguistique, voir Anne Trévise (dir.), «Activité et représentations métalinguistiques dans les acquisitions des langues », numéro spécial de AILE : Acquisition et interaction en langue étrangère, $1996, \mathrm{n}^{\circ} 8$. 
La perspective ouverte par les travaux sur l'intelligence multiple87 permet d'affirmer qu'un écrivain - considéré en psychologie cognitive comme un scripteur expert - possède une intelligence verbale très développée. Or ce type d'intelligence semble être lié notamment à la conscience métalinguistique qui a également un niveau de développement très élevé chez un écrivain. Bien que la conscience métalinguistique soit propre à tout écrivain (en sa qualité de scripteur expert), les études concernant l'impact positif du bilinguisme sur la conscience métalinguistique de l'enfant amènent à penser qu'elle doit être particulièrement développée chez les écrivains plurilingues. Les études préliminaires des différents cas d'écrivains qui parlent et écrivent plusieurs langues nous amènent à distinguer plusieurs manifestations de conscience métalinguistique que nous passerons en revue.

\section{Apprentissages d'autres langues comme moteur de créativité}

Chez certains écrivains plurilingues, la fascination pour les langues les amène quelquefois à apprendre de nouvelles langues, parfois très éloignées de leur univers culturel de référence. Ainsi, Vassilis Alexakis, écrivain d'origine grecque et d'expression française, se lance dans l'apprentissage du sango, langue véhiculaire de la République centrafricaine.

L'écrivain brésilien João Guimarães Rosa pousse cette curiosité intellectuelle et linguistique encore plus loin en multipliant les découvertes linguistiques :

Je parle : le portugais, l'allemand; le français, l'anglais, l'espagnol, l'italien, l'esperanto, un peu de russe; je lis : le suédois, le néerlandais, le latin et le grec (mais accroché au dictionnaire); je comprends quelques dialectes allemands; j'ai étudié la grammaire : du hongrois, de l'arabe, du sanscrit, du lituanien, du polonais, du tupi, de l'hébreu, du japonais, du tchèque, du finlandais, du danois; j'ai un peu fureté à propos d'autres langues. MAIS TOUT CELA, MAL. Je pense qu'étudier l'esprit et le mécanisme des autres langues nous aide beaucoup à comprendre plus en profondeur la langue du pays. Mais je les étudie principalement par plaisir, par goût et pour me distraire 88 .

Si ces langues ne semblent pas intervenir directement lors de son écriture en portugais, elles stimulent fortement la créativité linguistique de Guimarães Rosa.

En effet, les écrivains de ce type ont besoin de puiser leur inspiration dans d'autres systèmes linguistiques, même s'ils n'utilisent qu'une seule langue pour leur écriture.

\section{Traduction d'autres écrivains}

La passion des langues et du langage peut motiver l'intense activité de traduction à laquelle se livraient certains écrivains plurilingues. Cela fut par exemple le cas de Prosper Mérimée, infatigable traducteur en français de Gogol, de Pouchkine et de Tolstoï...

Un autre exemple remarquable est Paul Celan qui traduisait vers l'allemand du russe, de l'anglais, du roumain, du portugais, de l'hébreu et du français 89 .

87. Howard Gardner, Les Intelligences multiples, Paris, Odile Jacob, 1996.

88. Lettre de João Guimarães Rosa à sa nièce < http://www.germinaliteratura.com.br/pcruzadas_guimaraesrosa_ ago2006.htm > (consulté en décembre 2017).

89. Alexis Nouss, «Dans la ruine de Babel : poésie et traduction chez Paul Celan», TTR : traduction, terminologie, rédaction, vol. IX, n 1, 1996, p. 18. 
Rainer Maria Rilke, qui composait sa propre poésie en allemand, en français, en italien et en russe, avait également une incessante activité de traduction, en traduisant en allemand de la poésie du français, de l'italien, du latin, du moyen haut allemand, de l'anglais, du danois, du suédois, du russe 90 .

Pessoa est un autre exemple flagrant de poète-traducteur passionné, et cela malgré le fait qu'il considérait la traduction de la poésie comme un acte impossible : «nenhuma traducção, suppondo que existe, pode dar conhecimento da obra em sua completa e verdadeira vida» [aucune traduction, à supposer qu'elle existe, ne peut donner une représentation d'une œuvre dans sa forme complète et authentique] ${ }^{91}$. Pessoa a traduit de la poésie de l'anglais, du français, de l'espagnol, du grec, de l'allemand, du russe, du japonais, du perse, de l'italien vers le portugais, mais aussi vers d'autres langues notamment vers l'anglais et le français. Il se désignait lui-même en tant que traducteur :

Profissão: A designação mais propria será "traductor", a mais exacta a de "correspondente estrangeiro em casas commerciaes". O ser poeta e escriptor não constitue profissão, mas vocação.

[Profession : la désignation la plus appropriée sera «traducteur», la plus exacte sera «le correspondant étranger dans des maisons commerciales». Être poète et écrivain ne constitue pas une profession, mais une vocation. $]^{92}$

On peut citer également Lluis Maria Todo, romancier, critique littéraire et professeur d'université, qui est aussi traducteur chevronné vers le catalan des œuvres de Gustave Flaubert, Honoré de Balzac, Michel Tournier et Guy de Maupassant93. C'est aussi le cas de Rabearivelo qui a traduit de nombreux poèmes de Paul Valéry en malgache, et de la poésie malgache en français 94 .

Pour ce type d'écrivains qui se consacrent beaucoup à la traduction d'autres auteurs, l'activité de traduction occupe une place d'une importance comparable à celle que tient la création de leurs propres œuvres 95 .

On a pu observer chez certains écrivains que la traduction, surtout celle de la poésie, peut dériver vers leur propre composition poétique, l'acte traductif préfigurant et stimulant l'acte créatif. On le voit chez Valéry par exemple. Ainsi, le premier vers des Bucoliques de

90. Jessica Wilker, «Rilke, le même et l'autre : à propos de plusieurs versions d'un "même" poème», dans Axel Gasquet et Modesta Suárez (dir.), Écrivains multilingues et écritures métisses. L’hospitalité des langues, op. cit., p. 53-68.

91. Fernando Pessoa, «A virtude principal da literatura - o não ser música», dans Archives ouvertes de Pessoa, <http://arquivopessoa.net/textos/2407> (consulté en décembre 2017). Notre traduction ici et plus loin.

92. Cité dans Claudia J. Fischer, «Auto-tradução e experimentação linguística na génese d'O Marinheiro de Fernando Pessoa», dans Pessoa Plural I (P./Spr. 2012), p. 1-69, ici : p. 3. Disponible en ligne à l'adresse suivante : < http:// www.brown.edu/Departments/Portuguese_Brazilian_Studies/ejph/pessoaplural/Issue1/PDF/I1A01.pdf > (consulté en décembre 2017).

93. Alessandra Ferraro, «"Traduit par l'auteur". Sur le pacte autotraductif», dans Alessandra Ferraro et Rainer Grutman (dir.), L'autotraduction littéraire. Perspectives théoriques, Paris, Garnier, 2016, p. 121-140, ici : p. 139. 94. Claire Riffard, «Rabearivelo traducteur ou l'effet boomerang», Études littéraires africaines, ${ }^{\circ} 34$, «Traductions postcoloniale», sous la direction de Dominique Chancé et Alain Ricard, 2012, p. 29-41.

95. Cette activité de traduction tout au long de leur vie est à distinguer de la traduction à laquelle s'exercent les jeunes écrivains plurilingues. Dans ce dernier cas, il s'agit d'une sorte d'exercice qui leur permet de pratiquer et de perfectionner leur outil linguistique. Une fois rompus à l'exercice d'écriture par le biais de la traduction, ils commencent alors à écrire leurs propres fictions. 
Virgile, que Valéry traduit «O Tityre, tandis qu'à l'aise sous le hêtre», est transféré quasiment à l'identique dans la première réplique de son Dialogue de l'Arbre (1943) : "Que fais-tu là Tityre, amant de l'ombre à l'aise sous ce hêtre?». Quelques vers plus bas, le vers traduit par Valéry comme «toi, tranquille à l'ombre» présente une frappante ressemblance homophonique avec le célèbre incipit de son Cimetière Marin : «Ce toit tranquille, où marchent des colombes $\gg 96$. Ce passage d'un acte traductif à l'acte créatif, qui semble assez fréquent pour des poètes-traducteurs ${ }^{97}$, doit encore être illustré par l'apport de nouveaux corpus.

\section{Invention d'une langue}

Il existe également des écrivains qui ont poussé leur fascination pour les langues et pour le langage jusqu'à créer leur propre langue. C'est dans une certaine mesure le cas de Joyce ${ }^{98}$, et c'est le cas de Frédéric Werst qui, dans son roman Ward (Ier et IIème siècles), a créé une langue «ward»99. Les tentatives de création de langues, qui viennent contrecarrer la tendance à la disparition des langues naturelles, sont nombreuses. Initialement réservée aux savants aux idées utopiques 100 , la création de nouvelles langues tend à se démocratiser de nos jours 101 .

\section{Réflexion métalinguistique développée}

Certains écrivains ont développé une réflexion plus ou moins importante sur la langue et le style à l'instar de Proust qui a affirmé que «les beaux livres sont écrits dans une sorte de langue étrangère 102 ». On attribue également une réflexion particulièrement pertinente sur l'acte créatif à Valéry dont les manuscrits et la correspondance révèlent d'ailleurs un plurilinguisme caché103. Un écrivain plurilingue, surtout celui qui a appris plusieurs langues dans son enfance, a très tôt une conscience aiguë des particularités inhérentes à chaque langue : aux différences phonologiques, morphologiques, syntaxiques et sémantiques. Arrivés à l'âge adulte avec

96. Antonio Lavieri, «Paul Valéry. Tradurre il mondo, tradurre la poesia», Testo a fronte, n $^{\circ} 18,1998$, p. 147-164. C'est nous qui soulignons.

97. Lors de sa conférence «La patte du lion, la main de Saint Jérôme : approches génétiques de la traduction/ approches traductologiques de la genèse textuelle» au Congrès mondial de traductologie (les 11-14 avril 2017, université de Nanterre), Maria Teresa Giaveri a évoqué plusieurs autres exemples de perméabilité entre ces deux processus.

98. À ce sujet, voir Daniel Ferrer, «Finnegans Wake ou la créativité multilingue», dans Olga Anokhina (dir.), Multilinguisme et créativité littéraire, op. cit., p. 109-114.

99. Voir Anne-Laure Rigeade, «Ne pas écrire dans la langue des autres : Frédéric Werst, Ward (Ier et IIe siècles)», dans S'exprimer dans la langue des autres : postures d'auteurs, Actes du colloque international, université Lille 3 , Maison de la Recherche, les 7-8 mars 2013 (sous presse).

100. Voir Umberto Eco, La Recherche de la langue parfaite dans la culture européenne, Paris, Points, 1997. La tradition d'inventeurs de langue est très ancienne. Rappelons à ce titre la langue macaronique, apparue en Italie au XVe siècle, qui justement s'employait à combiner les éléments provenant de langue latine avec ceux des langues régionales. Cf. Fausta Garavini et al., «Écriture critique et genre macaronique», Bulletin de l'Association d'étude sur l'humanisme, la réforme et la renaissance, $\mathrm{n}^{\circ} 15,1982$, p. 40-47.

101. Serena Danna, «Inventer une langue, c'est facile (et ça peut rapporter gros)!», Courrier international, le 8 février 2012.

102. Marcel Proust, Contre Sainte-Beuve, Paris, Gallimard, 1954, p. 297-298.

103. Antonietta Sanna, «Les langues des affects : le cas de Valéry», dans Olga Anokhina (dir.), Multilinguisme et créativité littéraire, op. cit., p. 115-125. 
dans leurs bagages deux ou plusieurs langues pratiquées intensément y compris dans leur processus d'écriture, ces auteurs expriment alors de manière explicite comment ils vivent et écrivent à la fois en plusieurs langues et entre les langues. Ce numéro de Genesis présente, sous forme d'entretiens inédits, les réflexions de deux écrivains plurilingues qui ont beaucoup réfléchi et écrit sur les langues, sur l'écriture, sur cet espace singulier «entre les langues»: Luba Jurgenson, d'origine russe (entretien avec Julia Holter) et Gao Xingjian, d'origine chinoise (entretien avec Simona Gallo). Il est intéressant de noter que les écrivains relèvent l'importance de leur âge au moment où ils ont quitté leur pays natal : ce moment - plus ou moins douloureux - opère une sorte de césure dans leur contact avec leur milieu linguistique d'origine et cristallise leur compétence linguistique. Ainsi, le russe de Luba Jurgenson s'est en quelque sorte figé à l'âge de 17 ans quand elle a quitté l'Union soviétique.

\section{Conclusion}

Les recherches sur le plurilinguisme littéraire font partie d'un domaine plus large qui étudie différentes manifestations du plurilinguisme. Il s'agit d'une discipline en pleine formation qui pourrait être appelée - à l'instar du champ interdisciplinaire apparu dans les années 1960-1970 et qui fut nommé sciences cognitives -, études du multilinguisme (multilingual studies). Il est fondamental d'y inclure, dès à présent, les recherches sur l'écriture plurilingue, qui s'appuient sur une méthodologie scientifique - la critique génétique - dont la solidité a fait ses preuves. C'est seulement en se fondant sur des données empiriques vérifiables fournies par les traces écrites dans les documents de travail que le domaine des études littéraires pourra éviter l'écueil des théories non fondées et s'imposer à côté d'autres disciplines dans ce nouveau champ de recherche interdisciplinaire.

L'approche génétique du plurilinguisme littéraire apporte en effet une précieuse contribution à la connaissance des corpus concernés sans courir le risque de diluer le plurilinguisme dans un vaste univers théorique et abstrait. Elle contribue également à l'intelligibilité d'un domaine parfois cantonné à quelques études exemplaires et qui doit encore s'affirmer face à la persistance des préjugés monolingues. Car, dans les contextes plurilingues, la situation de l'auteur ainsi que son style sont indissolublement liés à une diversité des idiomes et à une pratique originale de la traduction dont les traces sont visibles et vérifiables dans les archives. Cette approche génétique, qui renoue avec l'esprit historique et linguistique des études pionnières, permet de parachever la déconstruction des conceptions monolingues et monoculturelles de la littérature, en prouvant concrètement que le multiculturalisme et le plurilinguisme sont des éléments essentiels de la créativité littéraire. Elle est également susceptible d'étendre considérablement le domaine du plurilinguisme en montrant à quel point ce phénomène est prégnant dans toutes les littératures du monde.

Tout comme l'observation de la création collective ou celle des processus créatifs dans les domaines non littéraires (musique, architecture, théâtre, cinéma...), l'étude des écrivains plurilingues et des traducteurs enrichit considérablement les corpus explorés jusque-là par la critique génétique en y apportant de nouveaux questionnements et défis théoriques et méthodologiques. Si, comme l'ont montré les théoriciens et les études cités plus haut, aucune écriture n'est réellement monolingue, les écritures qui jouent sur plusieurs langues naturelles permettent aux généticiens de saisir des mécanismes qui restent habituellement imperceptibles. Dans la mesure où l'écriture plurilingue est étroitement liée à l'(auto)traduction 
et la traduction collaborative qui amènent toutes les deux à la recréation d'une œuvre dans une autre langue, ce numéro fait écho à deux autres problématiques - traduction et création collaborative - qui ont été mises en lumière ces dernières années par Genesis et auxquelles le prisme du plurilinguisme littéraire et du système littéraire mondial offre un éclairage singulier particulièrement pertinent.

Comme le montrent plusieurs cas déjà évoqués, on a pu ainsi mettre au jour l'importance du plurilinguisme chez des écrivains parfois réduits à une dimension uniquement nationale en révélant des aspects demeurés jusqu'alors inconnus de leurs œuvres et de leurs traductions. Finalement, il s'agit d'appliquer le programme d'Auerbach qui, à propos de la philologie de la littérature mondiale, affirmait : «notre patrie philologique est la terre; ce ne peut plus être la nation $104 »$.

104. Erich Auerbach, «Philologie de la littérature mondiale», traduit par Diane Meur, dans Christophe Pradeau et Tiphaine Samoyault (dir.), Où est la littérature mondiale?, Saint-Denis, Presses universitaires de Vincennes, 2005, p. 37. 
OLGA ANOKHINA est chercheur à l'Institut des Textes et Manuscrits Modernes (CNRS-ENS). Elle consacre ses recherches à la genèse des œuvres des écrivains plurilingues et à la relation entre le plurilinguisme et la créativité littéraire. Olga Anokhina s’intéresse également aux aspects cognitifs de la production écrite et de la création. À l'ITEM, elle dirige l'équipe «Multilinguisme, traduction, création». Elle a publié plusieurs recueils et ouvrages collectifs, dont Critique génétique : concepts, méthodes, outils (avec Sabine Pétillon, 2009); Multilinguisme et genèse des textes (avec Natalia Vélikanova et al., 2010); Multilinguisme et créativité littéraire (2012); Écrire en langues : littératures et plurilinguisme (avec François Rastier, 2015); Du texte à l'image : une genèse réinventée (avec Fatiha Idmhand, 2018).

olga.anokhina@ens.fr

Emilio Sciarrino est ancien élève de l'École normale supérieure, agrégé d'italien et docteur en études italiennes. Ses recherches portent sur le plurilinguisme littéraire et sur la traduction. Membre de l'équipe «Multilinguisme, traduction, création » de l'ITEM, il a codirigé le volume collectif La Traduction de textes plurilingues italiens, paru en 2015 aux Éditions des Archives Contemporaines. En 2016, il a publié l'essai Le Plurilinguisme en littérature. Le cas italien, chez le même éditeur.

sciarrino.emilio@gmail.com

Résumés

A près avoir rappelé les apports des études fondatrices sur le plurilinguisme littéraire, cet article montre que la critique génétique est un outil fondamental pour comprendre ce phénomène. L'étude des archives des écrivains, en particulier de leurs brouillons, consent en effet de prolonger et de nuancer les interprétations purement théoriques du plurilinguisme. En outre, la critique génétique permet d'identifier concrètement les différentes situations plurilingues des auteurs pour mieux cerner leurs principales stratégies d'écriture. Elle révèle enfin la continuité entre écriture créative et traduction, qui se manifeste notamment par des formes particulières comme l'autotraduction et la traduction collaborative.

We will first recount the contributions that founding studies brought to literary multilingualism and will then show how genetic criticism is a fundamental tool for understanding this phenomenon. Indeed, the study of writers' archives, specially their drafts, helps to extend and nuance the purely theoretical interpretations of multilingualism. Furthermore, genetic criticism allows us to significantly identify writers' various multilingual situations in order to better grasp their main writing strategies. Finally, genetic criticism reveals the continuity between creative writing and translation in their works, notably manifest in particular forms such as self-translation and collaborative translation.

Dieser Artikel erinnert zuerst an Beiträge grundlegender Studien zur literarischen Mehrsprachigkeit, bevor er zeigt, dass die critique génétique ein wesentliches Instrument ist, um diese Phänomene zu verstehen. Das Studium der Archive von Autoren, insbesondere ihrer Entwürfe, trägt dazu bei, die rein theoretischen Interpretationen der Mehrsprachigkeit auszudehnen und zu differenzieren. Darüber hinaus ermöglicht die critique génétique, die verschiedenen mehrsprachigen Situationen von Autoren konkret zu identifizieren sowie deren wichtigste Schreibstrategien besser zu erfassen. Sie zeigt schließlich die Kontinuität zwischen kreativem Schreiben und Übersetzen in den Werken auf, was sich besonders in bestimmten Formen wie der Selbstübersetzung und der gemeinsamen Übersetzung zeigt.

\section{Plurilinguisme littéraire : de la théorie à la genèse}

Después de haber evocado los aportes de los estudios fundadores acerca del plurilingüismo literario, este artículo muestra que la crítica genética es una herramienta fundamental para comprender este fenómeno. El estudio de los archivos de escritores -particularmente de sus borradores- permite en efecto ampliar y matizar las interpretaciones puramente teóricas del plurilingüismo. La crítica genética permite, además, identificar concretamente las diferentes situaciones plurilingües de los autores para definir mejor sus principales estrategias de escritura, revelando, finalmente, la continuidad entre escritura creativa y traducción en sus obras continuidad que se manifiesta sobre todo en formas particulares como la autotraducción y la traducción colaborativa.

Depois de relembrar a contribuição de estudos pioneiros sobre o plurilinguismo literário, o presente artigo mostra que a crítica genética constitui ferramenta essencial para a compreensão desse fenômeno. O estudo dos arquivos de escritores, especialmente de seus rascunhos, fornece subsídios para ampliar e matizar as interpretações puramente teóricas do plurilinguismo. Além disso, a crítica genética permite identificar concretamente as diferentes situações plurilíngues dos autores, de modo a melhor reconhecer suas principais estratégias de escrita. Finalmente, ela revela a continuidade existente entre escrita criativa e tradução nas suas obras, fato que se manifesta com maior evidência em formas como a autotradução e a tradução colaborativa.

Dopo aver ricordato gli apporti degli studi fondatori sul plurilinguismo letterario, quest'articolo mostra che la critica genetica è uno strumento fondamentale per capire meglio questo fenomeno. Lo studio degli archivi degli scrittori, in particolare dei loro "scartafacci", consente infatti di approfondire e di riconsiderare le interpretazioni puramente teoriche del plurilinguismo. Inoltre, la critica genetica permette d'identificare concretamente le diverse situazioni linguistiche degli autori per meglio mettere a fuoco le loro principali strategie di scrittura, svelando infine la continuità tra scrittura creativa e traduzione nelle loro opere, soprattutto nelle forme peculiari dell'autotraduzione e della traduzione collaborativa. 